Notfall Rettungsmed $2017 \cdot 20: 91-96$ DOI 10.1007/s10049-017-0286-6

๑) Springer Medizin Verlag GmbH 2017

CrossMark

\author{
B. W. Böttiger $\cdot$ F. Semeraro ${ }^{2} \cdot$ K.-H. Altemeyer ${ }^{3} \cdot$ J. Breckwold $^{4} \cdot$ U. Kreimeier ${ }^{5} \cdot$ \\ G. Rücker ${ }^{6} \cdot$ S. Wingen ${ }^{1}$ \\ ${ }^{1}$ Klinik für Anästhesiologie und Operative Intensivmedizin, Uniklinik Köln (AöR), Köln, Deutschland \\ ${ }^{2}$ Department of Anaesthesia and Intensive Care Medicine, Maggiore Hospital, Bologna, Italien \\ ${ }^{3}$ Saarbrücken, Deutschland \\ ${ }^{4}$ Dekanat der Medizinischen Fakultät, Universität Zürich, Zürich, Schweiz \\ ${ }^{5}$ Klinik für Anaesthesiologie, Klinikum der Universität München, München, Deutschland \\ ${ }^{6}$ Klinik und Poliklinik für Anästhesiologie und Intensivtherapie, Universitätsklinikum Rostock, Rostock, \\ Deutschland
}

\title{
KIDS SAVE LIVES - Schülerausbildung in Wiederbelebung
}

\section{Eine Erfolgsgeschichte für Deutschland und die Welt}

Der plötzliche Herz-Kreislauf-Stillstand außerhalb eines Krankenhauses ist die dritthäufigste Todesursache in der sog. zivilisierten Welt [1]. In Deutschland sterben so mehr als 50.000 Menschen im Jahr; in Europa und den USA sind es jeweils 300.000 bis 400.000 , die durch den Rettungsdienst reanimiert werden, letztendlich aber nicht bis zur Krankenhausentlassung überleben [2-5]. Die wichtigste Maßnahme für ein gutes Überleben ist der unmittelbare Start von kardiopulmonalen Reanimationsmaßnahmen (CPR) - in der Regel durch Laien, die einen solchen Herz-Kreislauf-Stillstand in mehr als $60 \%$ beobachten [6-9].

Nach einem plötzlichen Kreislaufstillstand mit Sistieren des Blutflusses kann das Gehirn für nur 3-5 min überleben. Der Rettungsdienst, auch wenn er wie bei uns sehr gut entwickelt ist, braucht in der Regel aber 6, 8, 10 und oft auch deutlich mehr Minuten bis er eintrifft [6]. Damit kommt der Rettungsdienst im Falle eines Herz-Kreislauf-Stillstands praktisch immer zu spät. Es ist gesichert, dass die frühe Initiierung von Reanimationsmaßnahmen durch Laien - wir sprechen hier auch vom „Zeitfenster für die Laienreanimation" - die Überlebensrate verdoppelt bis vervierfacht [6-9]. Damit ist die frühe Reanimation durch Laien sehr viel effektiver als jede andere therapeutische Intervention bei diesem Krankheitsbild. Die Telefonreanimation und die Ersthelfersysteme sind hier natürlich mit einzubeziehen. Dies wird in den aktuellen Leitlinien des European Resuscitation Council (ERC) zur kardiopulmonalen Reanimation auch entsprechend deutlich und prominent betont [10].

In Deutschland lag die Laienreanimationsrate bis vor wenigen Jahren noch konstant bei unter $20 \%$, und mittlerweile liegen wir bei über $30 \%$. In einigen wenigen europäischen Ländern liegt die Laienreanimationsrate dagegen bei $40-60 \%$ und in manchen sogar bei 70-80\% [6, $9,11]$. Hätten wir in Deutschland ähnliche Verhältnisse, so würden wir pro Jahr 10.000 Menschenleben zusätzlich retten.

Zur weiteren Steigerung der Laienreanimationsrate stehen verschiedene Möglichkeiten zur Verfügung: umfangreiche und nachhaltige - ggf. niedrigschwellige - mediale Aufklärungsaktivitäten, die leitstellenassistierte Telefonreanimation, weitere Reanimationsausbildung von Erwachsenen, die Reanimationsausbildung von Schulkindern, Ersthelfersysteme und vieles mehr $[5,9,10,12]$.

In verschiedenen Ländern hat sich sehr klar gezeigt, dass insbesondere die
Ausbildung von Schulkindern in Wiederbelebung mit einem Anstieg der Laienreanimationsrate und mit einem Anstieg des Überlebens assoziiert ist [9]. Wir wissen, dass ein solches Training mit 12 Jahren starten und mindestens $2 \mathrm{~h}$ pro Jahr betragen sollte, solange die Kinder bzw. Jugendlichen in die Schule gehen [12-17]. Ein solches Training ist sehr einfach und kosteneffektiv. Es kann sowohl durch medizinisches Fachpersonal als auch durch entsprechend ausgebildete Lehrkräfte durchgeführt werden [12-14, 17-20]. Ein gutes Konzept wäre es, wenn zukünftige Lehrkräfte das schon in ihrem Studium lernen würden, wie das in Norwegen bereits der Fall ist. Das Training von Schulkindern in Wiederbelebung erhöht dabei auch spürbar die soziale Interaktion und die soziale Kompetenz, und fast immer ist es auch mit sehr viel Spaß für alle Beteiligten verbunden $[14,16]$. Schulkinder können dabei sehr gut auch als Multiplikatoren dienen, wenn man ihnen die Hausaufgabe gibt, in den folgenden 2 Wochen 10 weiteren Menschen zu zeigen, wie man reanimiert [14].

In Europa ist die Schülerausbildung in Wiederbelebung mittlerweile bereits in 5 Ländern gesetzlich verankert, in 16 weiteren - wie in Deutschland und in der 


\section{European Education CPR Map}

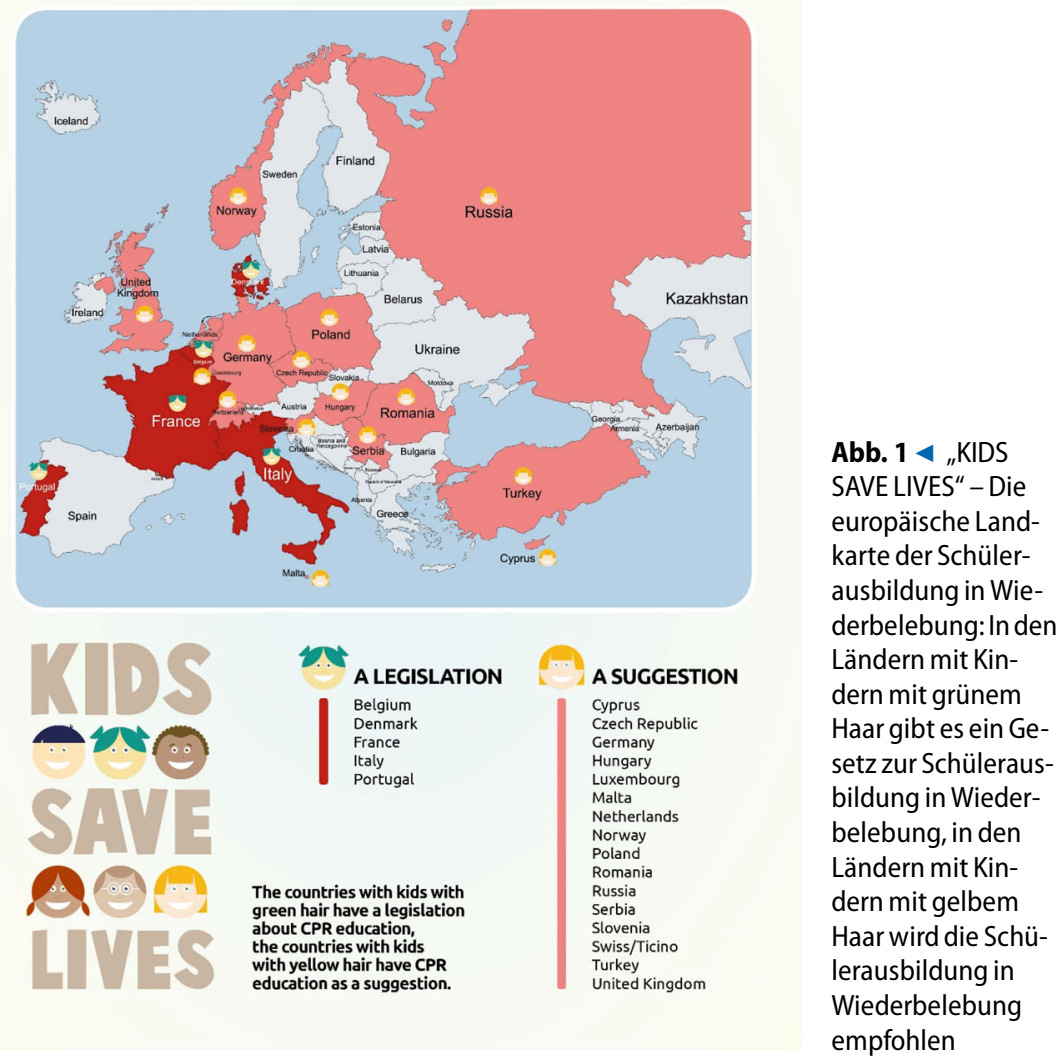

Schülerinnen und Schüler werden, gemeinsam mit dem Verein miniSanitäter aus München, im Kölner Kongresszentrum Gürzenich vor laufenden Kameras aller relevanten deutschen Fernsehsender in Wiederbelebung ausgebildet: http://www. minisanitaeter.de/projekte5.html.

2010

- Anästhesisten der Uniklinik Rostock initiieren in MecklenburgVorpommern - mit Unterstützung des dortigen Schulministeriums und der Björn-Steiger-Stiftung - die landesweite Ausbildung von Lehrkräften in Wiederbelebung mit dem Ziel, alle Schülerinnen und Schüler durch diese Lehrer entsprechend ausbilden zu lassen.

2011

- Vertreter von ERC und GRC starten im EU-Parlament in Straßburg eine Initiative zur Wiederbelebung, auch zum Schulunterricht in Wiederbelebung („Written Declaration on Establishing a European Cardiac Arrest Awareness Week “), die von 400 EU-Parlamentariern - und damit von einer Mehrheit der Abgeordneten aller Parteien im EU-Parlament unterschrieben und unterstützt wird.

\section{2} im vergangenen Jahrhundert. In der Gründungsversammlung des Deutschen Rates für Wiederbelebung/German Resuscitation Council (GRC) wurde 2007 ein interdisziplinärer Arbeitskreis zur Schülerausbildung initiiert. Gleichzeitig gab es Initiativen und Projekte in den Städten und Regionen Aachen und Münster (2006-2012: Langzeitstudie an Schulen, 2 Stunden Unterricht pro Jahr durch Lehrerinnen und Lehrer ist ausreichend) sowie in Rostock, Saarbrücken (seit ca. 1990 Aktivitäten an Schulen), u. a. [12, 13, 17].

\section{9}

hier Norwegen, wo seit Jahrzehnten Schülerinnen und Schüler in Wiederbelebung ausgebildet werden, und Dänemark, das im Rahmen
- Kongress des GRC und des ERC an der Uniklinik in Köln - 400
- Der GRC erstellt interdisziplinär und interprofessionell das „GRC Ausbildungskonzept für einen Reanimationsunterricht innerhalb der Schul-Curricula in Deutschland", es wird 2012 bzw. 2013 in deutscher und in englischer Sprache veröffentlicht. Dieses GRC Ausbildungscurriculum wird gemeinsam erarbeitet und getragen von allen im GRC vertretenen Organisationen und Hilfsorganisationen (ArbeiterSamariter-Bund [ASB], Bundesvereinigung der Arbeitsgemeinschaften der Notärzte Deutschlands [BAND], Deutscher Berufsverband Rettungsdienst [DBRD], Deutsche Gesellschaft für Anästhesiologie und 


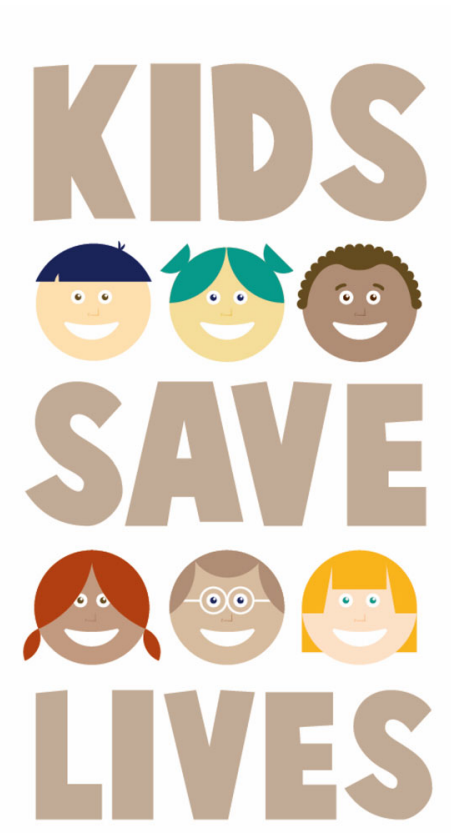

Abb. $2 \Delta$ Die internationale „KIDS SAVE LIVES"-Initiative wird durch dieses Logo repräsentiert, das vom Italienischen Rat für Wiederbelebung/Italian Resuscitation Council (IRC) erstellt wurde. Das IRC erlaubt ausdrücklich die kostenlose und möglichst breite Verwendung und Verbreitung dieses Logos für alle „KIDS SAVE LIVES"-Initiativen, Aktivitäten und Kampagnen weltweit

Intensivmedizin [DGAI], Deutsche Gesellschaft für internistische Intensivmedizin und Notfallmedizin [DGIIN], Deutsche Gesellschaft für Kardiologie - Herz- und Kreislaufforschung [DGK], Deutsche Gesellschaft für Unfallchirurgie [DGU], Deutsche Lebens-RettungsGesellschaft [DLRG], Gesellschaft für Neonatologie und pädiatrische Intensivmedizin [GNPI], Deutsches Rotes Kreuz [DRK], Johanniter-Unfall-Hilfe [JUH], Malteser Hilfsdienst [MHD]): www.grc-org.de.

- Vertreter von ERC und GRC besuchen auch zum Thema Schülerausbildung in Wiederbelebung den EU-Kommissar für Gesundheit im Europäischen Parlament in Straßburg und erörtern Möglichkeiten der Unterstützung.
- Der erste, europaweite „European Restart a Heart Day" des ERC mit Aktionen in mehr als 20 europäischen Ländern - hat das Motto „CHILDREN SAVING LIVES“: www.erc.edu.

- Unter der Schirmherrschaft des damaligen Bundesgesundheitsministers Daniel Bahr wird in Deutschland die erste „Woche der Wiederbelebung“ durchgeführt - initiiert von der DGAI, dem Berufsverband Deutscher Anästhesisten (BDA), dem GRC und der Stiftung Deutsche Anästhesiologie. Die „Woche der Wiederbelebung" findet in diesem Jahr vom 18.-24.09.2017 bereits zum 5. Mal statt: www.einlebenretten.de.

- Vertreter von ERC und GRC zeigen gemeinsam mit dem 12-jährigen $\mathrm{Nic}$ und der 16-jährigen Kea, die 2012 Nic in einer Schule in Hennef/Sieg bei Köln erfolgreich wiederbelebt hat dem EU-Kommissar für Gesundheit und seinen Kolleginnen und Kollegen im Sitz der Europäischen Kommission in Brüssel vor laufenden Kameras, wie man wiederbelebt: www.erc.edu.

- Guinness Weltrekord der Klinik für Anästhesiologie und Operative Intensivmedizin in Münster: 11.840 Schülerinnen und Schüler werden auf dem Schlossplatz in Münster in Wiederbelebung ausgebildet: https://www.youtube.com/watch? $\mathrm{v}=\mathrm{j} 3 \mathrm{IVbYXXegc}$.

\section{4}

- Der Schulausschuss der Deutschen Kultusministerkonferenz empfiehlt nach einer Initiative von DGAI, GRC und aufgrund der Ergebnisse der flächendeckenden Ausbildung in Mecklenburg-Vorpommern den Bundesländern, Lehrkräfte zu qualifizieren, damit diese Schülerinnen und Schüler in Wiederbelebung ausbilden $-2 \mathrm{~h}$ pro Jahr ab der Jahrgangsstufe 7 : www.grc-org.de.

- Der GRC adaptiert das bereits 2012 an den Empfehlungen der deutschen Kultusministerkonferenz ausgerichtetes „GRC Ausbildungskonzept für einen Reanimationsunterricht innerhalb der Schul-Curricula in Deutschland“: www.grc-org.de.

- Die Deutsche Anästhesiologie und der GRC starten - in NordrheinWestfalen gemeinsam - in mehreren Bundesländern Initiativen in Richtung Politik zur Einführung der Schülerausbildung in Wiederbelebung: www.einlebenretten.de.

- Die European Patient Safety Foundation (EPSF), der ERC, das International Liaison Committee on Resuscitation (ILCOR) und die World Federation Of Societies of Anaesthesiologists (WFSA) unterstützen das „KIDS SAVE LIVES“Statement, welches $2 \mathrm{~h}$ Unterricht in Wiederbelebung pro Jahr ab der 7. Klasse empfiehlt $[15,16]$.

\section{5}

- Die Weltgesundheitsorganisation (WHO) „endorsed“ das „KIDS SAVE LIVES“-Statement von ERC, ILCOR, WFSA und EPSF, begrüßt es damit und unterstützt das vorgeschlagene Konzept weltweit $[15,16]$.

- Der sudanesische Gesundheitsminister erklärt seine Unterstützung bei der „KIDS SAVE LIVES“-Initiative.

- Die Schülerausbildung in Wiederbelebung ist Bestandteil der Europäischen Reanimationsleitlinien, die der ERC am 15.10.2015 in der 6. überarbeiteten Auflage veröffentlicht [22].

- In Italien setzt die Schulministerin ein Gesetz in Kraft, das die Schülerausbildung in Wiederbelebung landesweit vorsieht; der italienische Rat für Wiederbelebung/Italien Resuscitation Council (IRC), ERC und GRC sind vor Ort und unterstützen den Event mit einem Flashmob mit Schülerinnen und Schülern sowie bei der gemeinsamen Pressekonferenz im italienischen Parlament (• Abb. 2); http://www.resuscitationjournal. com/article/S0300-9572(16)30299-4/ abstract. 
2016

- In Frankreich werden Erste Hilfe und Wiederbelebung per Gesetz zum Pflichtunterricht in Schulen.

- Griechenland beteiligt sich an der „KIDS SAVE LIVES“-Bewegung: In Thessaloniki zeigen 300 Schülerinnen und Schüler, wie man wiederbelebt: https://m.youtube.com/watch? $\mathrm{v}=78$ AhihA9uHE.

- Der ERC veröffentlicht sein „KIDS SAVE LIVES“-Position-Statement [14]; das ERC Statement wird vielfach übersetzt und ist derzeit in 12 Sprachen auf der Homepage des ERC verfügbar. IRC, GRC und ERC veröffentlichen kurze Zeit später das „KIDS SAVE LIVES“-Video "Saving a life is a child's play", in dem die 10 Prinzipien des ERCPosition-Statements verfilmt sind; dieses Video steht ausdrücklich allen zur freien Verfügung und darf auf allen Kanälen verbreitet und geteilt werden: https://www.youtube.com/ watch? $=0$ Yf4umHnD3c.

- Die European Society for Anaesthesiology (ESA) unterstützt durch Publikation des ERC-Position-Statements-im ESA Newsletter die „KIDS SAVE LIVES“-Initiative: https:// www.esahq.org/resources/resources/ quarterly-esa-newsletter.

- IRC, GRC und ERC führen im Rahmen des „ERC Reserch NET“ eine detaillierte Umfrage zum Umsetzungsstatus der Schülerausbildung in Wiederbelebung in 34 europäischen Ländern durch und veröffentlichen die Ergebnisse [21].

- Der rumänische Gesundheitsminister unterstützt die weltweite „KIDS SAVE LIVES“-Initiative bei der WHO.

- In Deutschland wird unter der Schirmherrschaft von Bundesgesundheitsminister Hermann Gröhe und gemeinsam mit der Bundeszentrale für gesundheitliche Aufklärung (BZgA), mit dem GRC und zahlreichen anderen Fachgesellschaften und Organisationen das „Nationale Aktionsbündnis Wiederbelebung“ (NAWIB) gegründet. Bei der Auftaktveranstaltung des NAWIB vor dem Brandenburger Tor - mit Herrn Bundesgesundheitsminister Hermann Gröhe, der Leiterin der BZgA Frau Dr. Heidrun Thaiss, Herrn Dr. Eckart von Hirschhausen und Vertreterinnen und Vertretern aller beteiligten Fachgesellschaften und Organisationen - werden mehr als 100 Schülerinnen und Schüler in Wiederbelebung ausgebildet Kea und Nic sind mit dabei: www. wiederbelebung.de.

- Guinness Weltrekord der Uniklinik Köln - Menschen aus 74 Nationen führen ohne Unterbrechung an einer Puppe die Herzdruckmassage durch

- Kea ist mit dabei: https://youtu.be/ 83vCRWvw_lY.

- Der „European Restart a Heart Day“ 2016 des ERC - mit entsprechenden Aktionen in mehr als 20 europäischen Ländern - hat das Motto „KIDS SAVE LIVES“: www.erc.edu.

- Bundesgesundheitsminister Hermann Gröhe trainiert - gemeinsam mit Frau Dr. Heidrun Thaiss, der Leiterin der BZgA, Gerald Asamoah und Anästhesistinnen und Anästhesisten der Uniklinik Köln in der Gesamtschule Meiersheide in Hennef/Sieg - der Schule von Nic und Kea - 200 Schülerinnen und Schüler in Wiederbelebung: www.wiederbelebung. de.

\section{7}

Und wie geht es in diesem Jahr weiter?

- Die "Woche der Wiederbelebung“ unter der Schirmherrschaft von Bundesgesundheitsminister Hermann Gröhe wird in diesem Jahr vom 18.-24. September stattfinden.

- Vom 28.-30. September findet in Freiburg im Breisgau der Europäische Reanimationskongress „Resuscitation 2017“ des ERC statt; am Donnerstag, dem 28.09. gibt es dort den Satellitenkongress des GRC mit einer großen Aktion zu „KIDS SAVE LIVES“: www.grc-org.de.

\section{Fragen zur Umsetzung}

Vielleicht stellen Sie sich jetzt die Fragen:

- Was brauchen wir an Konzept bzw. an Ausstattung?

- Wie können wir möglichst einfach anfangen?

- Welche finanziellen Unterstützungen und Möglichkeiten gibt es?

Bis heute wurden in Deutschland verschiedene Methoden und Modelle für die Schülerausbildung in Wiederbelebung entwickelt und praktiziert - die alle auch miteinander kombiniert werden können $[14,19]$

\section{Trainingsmaterial}

- Kleine Trainingspuppen, die die Schülerinnen und Schüler auch mit nach Hause nehmen können und sollen (Multiplikationseffekt),

- „High-fidelity“-Trainigspuppen mit Feedback-Systemen für Schülergruppen.

\section{Wer unterrichtet?}

- Unterricht durch die Hilfsorganisationen,

- Unterricht durch Ärzte oder weiteres medizinisches Personal, ggf. unterstützt durch Fachgesellschaften ganz besonders engagiert sind hier die deutschen Anästhesisten,

- Unterricht durch entsprechend ausgebildete Lehrkräfte,

- Unterricht durch andere Schülerinnen und Schüler, insbesondere durch Schulsanitätsdienste.

\section{Unterrichtsformen}

- Training im erweiterten Rahmen von Biologie- und/oder Sportunterricht,

- Das Training kann im Rahmen von Vertretungsunterricht, Projekttagen/ -wochen und/oder anderen Schulund Sportveranstaltungen durchgeführt werden,

- Nutzung neuer Medien, um die Grundlagen der Wiederbelebung auf spielerische Art und Weise zu erlernen (Apps, Spiele, z. B. „A breathtaking picnic/Ein atemberaubendes 
Picknick“ https://www.youtube.com/ watch?v=UYlvdUcGjz0),

- Social-Media-Aktivitäten zur Steigerung der Aufmerksamkeit des Themas sowie auch zu medialen Verbreitung der eigenen Aktivitäten bei der Schülerausbildung in Wiederbelebung.

Mecklenburg-Vorpommern war mit dem Anästhesisten Gernot Rücker und anderen in Deutschland mit weitem Abstand der Vorreiter einer landesweiten Schülerausbildung in Wiederbelebung, danach kamen Baden-Württemberg, Sachsen und jetzt bald auch NordrheinWestfalen, Schleswig-Holstein, Niedersachsen und andere. Der Fokus der Schülerausbildung in Wiederbelebung liegt sinnvollerweise auf der Herzdruckmassage, denn diese reicht aufgrund des verbleibenden Sauerstoffs im Blut außerhalb des Gehirns zur Überbrückung bis der Rettungsdienst kommt meist aus. Auf das Training von Beatmung oder gar der Benutzung von automatisierten Defibrillatoren wird - entsprechend unserem Motto „PRÜFEN - RUFEN DRÜCKEN“ - in der Regel zunächst verzichtet [14].

\section{Finanzierungskonzepte}

Die Finanzierung der Schülerausbildung in Wiederbelebung ist in Deutschland leider bisher weder gesetzlich noch durch Erlass geregelt. Dies stellt Schulen vor eine große Herausforderung, denn für die Finanzierung müssen somit meistens lokale Lösungen gefunden werden: Unterstützung durch lokale Sponsoren, Elterninitiativen, Schulvereine, Krankenhäuser und Hilfsorganisationen u.v.a. mehr. Und es gibt auch überregionale Finanzierungskonzepte wie in BadenWürttemberg: Hier wird die Schülerausbildung in Wiederbelebung u.a. mit finanzieller Unterstützung der Landesregierung durchgeführt: http://loewenretten-leben.de/.

\section{Das Wichtigste}

Wir wissen, dass der Beginn einer Wiederbelebung durch Laien die effektivste Maßnahme zur Erhöhung des Überle- bens nach Herz-Kreislauf-Stillstand ist [6-9]. Die Ausbildung von Schülerinnen und Schülern - auch als Multiplikatoren - spielt hier eine ganz zentrale Rolle für eine schnelle und nachhaltige Erhöhung der Laienreanimationsrate und damit der Überlebensrate [14]. Nachdem trotz entsprechend klarer und einstimmiger Empfehlungen der Deutschen Kultusministerkonferenz von 2014 die Umsetzung der Schülerausbildung in Wiederbelebung aus verschiedenen Gründen bisher nur sehr schleppend verläuft, sollte diese auch bei uns am besten bundesweit gesetzlich verankert und gleichzeitig auch in allen Bundesländern flächendeckend implementiert werden.

Bis dahin können wir alle, können Sie etwas tun. Fangen Sie einfach an, helfen Sie einfach mit, machen Sie einfach etwas. Es wird bestimmt gut und macht Spaß. Weitere Informationen, Videos, Vorträge und Konzepte finden Sie z. B. unter den folgenden Links:

$$
\begin{aligned}
& \text { - www.grc-org.de } \\
& \text { - www.einlebenretten.de } \\
& \text { - www.wiederbelebung.de } \\
& \text { - www.bayernreanimiert.de } \\
& \text { - www.erc.edu } \\
& \text { - https://www.youtube.com/watch? } \\
& \text { v=0Yf4umHnD3c } \\
& \text { - https://www.youtube.com/watch? } \\
& \text { v=RsArHyybeWk } \\
& \text { - https://www.youtube.com/watch? } \\
& \text { v=VOoW4pGQhBM } \\
& \text { - https://youtu.be/LSNgOP5ujmQ } \\
& \text { - https://www.youtube.com/watch? } \\
& \text { v=UYlvdUcGjz0 }
\end{aligned}
$$

\section{Korrespondenzadresse}

Prof. Dr. B. W. Böttiger, M.L., D.E.A.A.,

F.E.S.C., F.E.R.C.

Klinik für Anästhesiologie und Operative Intensivmedizin, Uniklinik Köln (AöR)

Kerpener Straße 62, 50937 Köln, Deutschland

bernd.boettiger@uk-koeln.de

Prof. Dr. B. W. Böttiger ist Vorstandsvorsitzender des Deutschen Rates für Wiederbelebung (GRC), Board Director Science and Research, European Resuscitation Council (ERC), Mitglied in der „Advanced Life Support" (ALS) Task Force des International Liaison Committee on Resuscitation (ILCOR), Präsidiumsmitglied, Deutsche interdisziplinäre Vereinigung für Intensiv- und Notfallmedizin (DIVI), Direktor der Klinik für Anästhesiologie und Operative Intensivmedizin
Danksagung. Wir danken allen Kolleginnen und Kollegen, Ministerinnen und Ministern, Politikerinnen und Politikern, Menschen, Organisationen, Fachgesellschaften und Firmen sehr herzlich, die zu dieser äußerst positiven und wichtigen Entwicklung in Deutschland, in Europa und in der Welt beigetragen haben und auch denen, die uns in Zukunft weiter bei unserer KIDS SAVE LIVES Initiative unterstützen. Bernd Böttiger wird vom ERC Research NET unterstützt.

Interessenkonflikt. B. W. Böttiger ist European Resuscitation Council (ERC) Board Director Science and Research; Vorstandsvorsitzender des Deutschen Rates für Wiederbelebung/German Resuscitation Council (GRC); Mitglied in der "Advanced Life Support" (ALS) Task Force des International Liaison Committee on Resuscitation (ILCOR); Mitglied im Präsidium der Deutschen Interdisziplinären Vereinigung für Intensiv- und Notfallmedizim (DIVI); Associated Editor des European Journal of Anaesthesiology (EJA); Mitherausgeber der Zeitschrift Resuscitation; Schriftleiter der Zeitschrift Notfall + Rettungsmedizin. Für Vorträge hat er Honorare der folgenden Firmen erhalten: Medupdate, Forum für Medizinische Fortbildung (FoMF), Baxalta, Bayer Vita, ZOLL, BARD. F. Semeraro ist Vorsitzender des Italian Resuscitation Council (IRC). K.-H. Altemeyer, J. Breckwold, U. Kreimeier, G. Rücker und S. Wingen geben an, dass kein Interessenkonflikt besteht.

\section{Literatur}

1. Taniguchi D, Baernstein A, Nichol G (2012) Cardiac arrest: a public health perspective. Emerg Med Clin North Am 30:1-12

2. Mozaffarian D, BenjaminEJ, Go AS, ArnettDK, Blaha MJ, Cushman M, Das SR, de Ferranti S, Després JP, Fullerton HJ, Howard VJ, Huffman MD, Isasi CR, Jiménez MC, Judd SE, Kissela BM, Lichtman JH, Lisabeth LD, Liu S, Mackey RH, Magid DJ, McGuire DK, Mohler ER 3rd, Moy CS, Muntner P, Mussolino ME, Nasir K, Neumar RW, Nichol G, Palaniappan L, Pandey DK, Reeves MJ, Rodriguez CJ, Rosamond W, Sorlie PD, Stein J, Towfighi A, Turan TN, Virani SS, Woo D, Yeh RW, Turner MB, American Heart Association Statistics Committee, Stroke Statistics Subcommittee (2016) Executive summary: heart disease and stroke statistics - 2016 update: a report from the American Heart Association. Circulation 133:447-454

3. Böttiger BW, Semeraro F, Wingen S (2017) KIDS SAVE LIVES - educating schoolchildren in CPR - a civic duty that needs further support for implementation! J Am Heart Assoc. DOI:10.1161/ JAHA.117.005738

4. http://www.nap.edu/catalog/21723/strategiesto-improve-cardiac-arrest-survival-a-time-toact. Zugegriffen: 17. Februar 2017

5. Böttiger BW (2015) „A time to act" - anaesthesiologists in resuscitation help save 200,000 lives per year worldwide: school children, lay resuscitation, telephone-CPR, IOM and more. Eur J Anaesthesiol 32:825-827

6. Böttiger BW, Grabner C, Bauer H, Bode C, Weber T, Motsch J, Martin E (1999) Long term outcome after out-of-hospital cardiac arrest with physician staffed emergency medical services: the Utstein style applied to a midsized urban/suburban area. Heart 82:674-679

7. Breckwoldt J, Schloesser S, Arntz HR (2009) Perceptions of collapse and assessment of cardiac 
arrest by bystanders of out-of-hospital cardiac arrest (OOHCA). Resuscitation 80:1108-1113

8. Holmberg M, Holmberg S, Herlitz J (2000) Effect of bystander cardiopulmonary resuscitation in out-of-hospital cardiac arrest patients in Sweden. Resuscitation 47:59-70

9. Wissenberg $M$, Lippert FK, Folke F, Weeke $P$, Hansen $\mathrm{CM}$, Christensen $\mathrm{EF}$, Jans $\mathrm{H}$, Hansen PA, Lang-Jensen T, Olesen JB, Lindhardsen J, Fosbol EL, Nielsen SL, Gislason GH, Kober L, Torp-Pedersen C (2013) Association of national initiatives to improve cardiac arrest management with rates of bystander intervention and patient survival after out-of-hospital cardiac arrest. JAMA 310:1377-1384

10. Perkins GD, Handley AJ, Koster RW, Castrén M, Smyth MA, Olasveengen T, Monsieurs KG, Raffay V, Gräsner JT, Wenzel V, Ristagno G, Soar $J$ (2015) Basismaßnahmen zur Wiederbelebung Erwachsener und Verwendung automatisierter externer Defibrillatoren. Kapitel 2 der Leitlinien zur Reanimation 2015 des European Resuscitation Council. NotfRettungsmed 18:748-769

11. Gräsner JT, Bossaert L (2013) Epidemiology and management of cardiac arrest: what registries are revealing. Best Pract Res Clin Anaesthesiol 27:293-306

12. Rücker G, Schubert J, Scheeren T, NöldgeSchomburg G (2010) Wiederbelebungsunterricht bei Schülern: Ab der siebten Klasse sinnvoll, Dtsch Arztebl 107: A-492/B-430/C-422

13. Bohn A, Lukas RP, Breckwoldt J, Böttiger BW, Van Aken H(2015) „Kids save lives": why schoolchildren should train in cardiopulmonary resuscitation. Curr Opin Crit Care 21:220-225

14. Böttiger BW, Bossaert LL, Castrén M, Cimpoesu $D$, Georgiou M, Greif R, Grünfeld M, Lockey A, Lott C, Maconochie I, Melieste R, Monsieurs KG, Nolan JP, Perkins GD, Raffay V, Schlieber J, Semeraro F, Soar J, Truhláŕ A, Van de Voorde P, Wyllie J, Wingen $\mathrm{S}$, Board of European Resuscitation Council (ERC) (2016) Kids Save Lives - ERC position statement on school children education in CPR: „Hands that help - Training children is training for life". Resuscitation 105:A1-A3

15. Böttiger BW, Van Aken H(2015) Training children in cardiopulmonary resuscitation worldwide. Lancet 385:2353

16. Böttiger BW, Van Aken H (2015) Kids save lives - Training school children in cardiopulmonary resuscitation worldwide is now endorsed by the World Health Organization (WHO). Resuscitation 94:A5-A7

17. Cave DM, Aufderheide TP, Beeson J et al (2011) Importance and implementation of training in cardiopulmonary resuscitation and automated externaldefibrillation in schools: ascienceadvisory from the American Heart Association. Circulation 123:691-706

18. DeBuckE, Van Remoortel H,Dieltjens T, Verstraeten $\mathrm{H}$, Clarysse M, Moens O, Vandekerckhove P (2015) Evidence-based educational pathway for the integration of first aid training in school curricula. Resuscitation 94:8-22

19. Lukas RP, Van Aken H, MölhoffT, Weber T, Rammert M, Wild E, Bohn A (2016) Kids save lives: a sixyear longitudinal study of schoolchildren learning cardiopulmonary resuscitation: Who should do the teaching and will the effects last? Resuscitation 101:35-40

20. Plant N, Taylor K (2013) How best to teach CPR to schoolchildren: a systematic review. Resuscitation 84:415-421
21. Semeraro $F$, Wingen $S$, Schroeder DC, Ecker $H$, Scapigliati A, Ristagno G, Böttiger BW (2016) KIDS SAVE LIVES implementation in Europe: a survey through the ERC Research NET. Resuscitation 107:e7-e9. doi:10.1016/j.resuscitation.2016.08. 014

22. Greif R, Lockey AS, Conaghan P, Lippert A, De Vries W, Monsieurs KG (2015) Ausbildung und Implementierung der Reanimation. Kapitel 10 der Leitlinien zur Reanimation des European Resuscitation Council. Notf Rettungsmed 18:1016-1034
Rettungsdienste rüsten sich wegen Terrorgefahr

Um im Fall eines Anschlags gewappnet zu sein, verändern mehrere Bundesländer die Anforderungen an ihre Rettungsdienste.

Bayern, Baden-Württemberg und Berlin haben die Ausrüstung von Rettungswagen ergänzt oder planen dies, wie eine Umfrage der Deutschen Presse-Agentur ergab. Berlin rüstete bereits 2013 seine Rettungswagen auf, Bayern tat das im Juni. Baden-Württemberg erarbeitet derzeit ein Konzept für eine erweiterte Ausrüstung. In Hessen will man an der Ausrüstung nichts verändern, hat aber eine Arbeitsgruppe von Ministerien und Hilfsorganisationen gebildet. Viele der neuen Ausrüstungsgegenstände werden bisher vor allem beim Militär eingesetzt, etwa Tourniquets zum schnellen Abbinden stark blutender Gliedmaßen. Diese könnten zum Beispiel nach einer Bombenexplosion zum Einsatz kommen. Bayern führte zudem Wundverbände ein, die durch einen Wirkstoff lebensbedrohliche Blutungen von sich aus stillen. In Berlin kamen neben Tourniquets spezielle blutungsstillende Medikamente neu auf die Fahrzeuge. Alle übrigen Bundesländer fühlen sich bereits ausreichend auf einen Anschlag vorbereitet. In einigen, etwa dem Saarland, Bremen und Hamburg, gehören zumindest Tourniquets bereits seit längerem zur Ausstattung der Rettungswagen. Auch für Krankenhäuser wird die Vorbereitung auf Terrorlagen immer wichtiger. Beispielsweise in Hannover wurde bereits zur Fußball-WM 2006 ein Notfallplan aufgestellt, in dem auch geregelt ist, wie sich Kliniken gegenseitig entlasten können. Zudem hat sich ein Markt für Fortbildungen zum Thema Terror gebildet. Das DRK schult seine Mitarbeiter etwa auf die Gefahr eines "Zweitschlags" hin. Um der Gefahr von Anschlägen zu begegnen, beschloss der Bund die Aufstellung von 61 Medizinischen Task Forces (MTF). Diese Verbände mit Ärzten, Sanitätern und Gerätewagen sollen bei Massenanfall von Verletzten die Versorgung länderübergreifend sicherstellen. Der Aufbau sei zu 80 Prozent abgeschlossen.

Quelle: Ärzte Zeitung www.aerztezeitung.de 